\title{
Corela
}

Cognition, représentation, langage

HS-8 | 2010

L'interpellation

\section{Interpeler l'absent.}

Le rôle de la convocation du disparu dans les nécrologies

\section{Marie-Laure Florea}

\section{(2) OpenEdition}

Journals

Édition électronique

URL : http://journals.openedition.org/corela/743

DOI : $10.4000 /$ corela.743

ISSN : 1638-573X

Éditeur

Cercle linguistique du Centre et de l'Ouest - CerLICO

\section{Référence électronique}

Marie-Laure Florea, « Interpeler l'absent. », Corela [En ligne], HS-8 | 2010, mis en ligne le 01 octobre 2010, consulté le 19 avril 2019. URL : http://journals.openedition.org/corela/743 ; DOI : 10.4000/ corela.743

Ce document a été généré automatiquement le 19 avril 2019

\section{(c) (1) (2)}

Corela - cognition, représentation, langage est mis à disposition selon les termes de la licence Creative Commons Attribution - Pas d'Utilisation Commerciale - Partage dans les Mêmes Conditions 4.0 International. 


\section{Interpeler l'absent.}

Le rôle de la convocation du disparu dans les nécrologies

\section{Marie-Laure Florea}

1 Je commencerai en esquissant une définition (provisoire) de l'interpellation : on peut dire que l'interpellation est un acte allocutif qui consiste à poser autrui comme interlocuteur par le biais de la forme même de son discours. La plupart du temps, ce destinataire est un être vivant, mais il peut aussi être inanimé ou absent, et c'est à ce type d'interpellation en marge de la notion que je vais me consacrer ici, puisqu'il s'agit d'analyser les interpellations du disparu dans les nécrologies de presse. Il s'agit donc là d'une première originalité du type d'interpellation que je me propose d'analyser, originalité qui concerne la nature de l'interpelé.

2 Mais ce type d'interpellation est également un phénomène original du fait de son caractère marginal au sein du genre nécrologique. En effet, la nécrologie prend habituellement la forme d'un récit hétérodiégétique dans lequel le disparu est délocuté : une nécrologie est écrite par un autre (on ne peut pas parler de sa propre mort, comme nous le rappelle opportunément la citation en exergue) et pour d'autres (la personne dont il est question ne lira jamais sa nécrologie). Ainsi, par la force du contexte, le disparu est habituellement exclu de la relation d'interlocution. Toutefois, cette situation de communication traditionnelle souffre parfois quelques entorses : il arrive que le défunt accède aux deux rôles qui lui sont traditionnellement inaccessibles, celui de locuteur et celui d'interlocuteur. C'est ce dernier cas que je souhaite analyser ici.

3 Je tiens à souligner que cette pratique qui consiste à interpeler le disparu dans une nécrologie, bien que marginale à double titre, comme je viens de le relever, est loin d'être anecdotique. Elle est en effet d'une part intéressante dans une perspective théorique : en tant précisément que phénomène marginal, elle peut nous permettre de mieux cerner les frontières de la notion d'interpellation. D'autre part, cette pratique est significative du fonctionnement de la nécrologie, qui, comme on le verra, vise à redonner corps à l'absent. Pour ces deux raisons, ce phénomène mérite la place que je me propose de lui accorder ici.

4 Je commencerai par donner un rapide aperçu de l'interpellation dans les nécrologies. Je tenterai ensuite de remodeler la définition de l'interpellation à la lumière de mon corpus, 
en la faisant sortir du cadre énonciatif bipolaire dans lequel elle est figée. Enfin, à partir de cette redéfinition du phénomène, je m'attacherai à déterminer le rôle tenu par l'interpellation du disparu dans les nécrologies.

\section{L'interpellation en contexte nécrologique}

\subsection{Modalités d'interpellation}

5 Assez classiquement, on trouve dans les nécrologies deux types de procédés interpellatifs : des procédés intrinsèquement interpellatifs et des procédés connexes. Les procédés intrinsèquement interpellatifs visent à interpeler l'allocutaire et se suffisent à eux-mêmes pour ce faire. C'est tout d'abord le cas de l'apostrophe, comme dans les deux exemples suivants :

(1) «Je t'aimais beaucoup, François, mon camarade des salles obscures » [LACHIZE Samuel, « Ô François... », L'Humanité, 15/05/06 : 22]

(2)« Salut l'artiste. » [SIRACH Marie-José, « Une sensibilité à l'autre authentique », France Soir, 19/06/06: 21]

6 Les termes d'adresse employés ici sont représentatifs de ce que l'on trouve dans les nécrologies : il s'agit le plus souvent de noms propres - généralement le prénom, plus rarement le nom - quelquefois expansés (comme dans l'exemple 1), parfois de noms de métiers.

7 Le second procédé intrinsèquement interpellatif est l'emploi du mode impératif, comme dans l'exemple suivant où l'auteur, qui a fréquenté le disparu de son vivant (tous deux étaient journalistes), l'exhorte à se remémorer les bons moments qu'ils ont partagés :

(3) «Ô François... [...] rappelle-toi du temps où Claude Chabrol, lorsqu'il avait terminé un film, nous invitait tous les trois (ses « cocos») dans une gargote de bon aloi dont il avait le secret.» [LACHIZE Samuel, « Ô François... ", L'Humanité, $15 / 05 / 06: 22]$

Par ailleurs, l'interpellation se fait également au moyen de ce que j'ai appelé des procédés d'interpellation connexes, c'est-à-dire qui sont toujours accompagnés d'une forme intrinsèquement interpellative. Il s'agit de l'emploi d'un pronom de deuxième personne (exemple 1), de la salutation (exemple 2), ou encore du remerciement :

(1) «Je t'aimais beaucoup, François, mon camarade des salles obscures » [LACHIZE Samuel, « Ô François... », L'Humanité, 15/05/06 : 22]

(2) « Salut l'artiste. » [SIRACH Marie-José, «Une sensibilité à l'autre authentique », France Soir, 19/06/06:21]

(4) «Merci, cher Georges-Paul.» [FIGUERAS Olivier, «À Dieu, Georges-Paul, et merci », Présent, 20/06/06: 4]

9 Ces formes sont, dans les nécrologies de mon corpus, toujours couplées à une apostrophe.

\subsection{Place dévolue à l'interpellation}

10 Afin de compléter cet inventaire des formes interpellatives, nous allons maintenant voir quelle place est dévolue à l'interpellation dans les nécrologies. On peut distinguer deux cas : la plupart du temps, l'interpellation n'a dans la nécrologie qu'une place marginale, l'article conservant une énonciation classique sur laquelle vient se greffer une forme d'interpellation. Cette séquence interpellative se situe habituellement aux frontières du texte, soit dans le titre (exemple 5), soit en position de clôture (exemple 6). 
(5) « T'es pas drôle Raymond ! » [STICO Bernie, « T'es pas drôle Raymond ! », France Soir, 16/06/06:24]

(6) «À Dieu, Devos! que votre verbe scintillant retentisse désormais, par-delà les étoiles, au paradis des anges. " [FIGUERAS Olivier, «Le plus grand des mots ", Présent, 17/06/06:2]

11 Dans de rares cas, il arrive que l'interpellation envahisse l'ensemble de l'article, la nécrologie étant alors adressée au défunt de bout en bout. Ce cas est toutefois exceptionnel : il ne s'est présenté que trois fois sur les quelque cinq cents nécrologies du corpus étudié. Ces trois cas sont toutefois fort différents les uns des autres : dans le premier, il s'agit d'une invocation du disparu :

(3) «Ô François... [...] rappelle-toi du temps où Claude Chabrol, lorsqu'il avait terminé un film, nous invitait tous les trois (ses « cocos») dans une gargote de bon aloi dont il avait le secret.» [LACHIZE Samuel, "Ô François... ", L'Humanité, 15/05/06 : 22]

Dans le second cas, l'interpellation revêt la forme d'une lettre au disparu, l'article lui étant par conséquent adressé dans son ensemble :

(7) «Mon cher Georges-Paul. J'ai peine à croire que j'écris ces lignes et que vous n'êtes plus là. [...] Vous arriviez au journal le jeudi matin bien avant l'aube avant d'aller au palais et c'était mon tour d'être le « chef du jour ». Que l'on juge bien de l'incongruité de la situation, c'était à moi de vous " commander» un article et de vous diriger ce jour-là. Cela vous faisait sourire avec une parfaite délicatesse, et ces moments-là ont été pour moi une formidable école. Nous avons travaillé, je le crois, avec beaucoup d'ardeur, de complicité, de bonheur.» [PARMENTIER Caroline, « Georges-Paul », Présent, 13/06/06:1]

13 Enfin, le dernier cas d'article intégralement interpellatif est constitué par cette nécrologie de Jean-Pierre Neu, parue dans les Échos :

(8) « Jean-Pierre est parti, hier, sur un grand bateau blanc. [...] Jean-Pierre, tu étais notre copain à tous, mon ami et un grand journaliste. [...] Ton rire, tes fêtes, ton muscat, tes scoops, ton appétit de la vie, ton amour des autres restent imprimés dans nos têtes et sur les murs de ce journal comme l'encre sur le papier.» [ESCANDE Philippe, « Jean-Pierre Neu », Les Échos, 17/05/06: 10]

14 Cette nécrologie est particulière dans le sens où elle ressemble, de par sa structure, à une nécrologie classique, à ceci près qu'elle est transposée à la deuxième personne. Elle diffère en cela des deux précédentes, qui ne pourraient être rétablies à la troisième personne comme une nécrologie traditionnelle, dans la mesure où les procédés interpellatifs sont au cœur même de la forme des articles, qui sont entièrement adressés au défunt, notamment grâce à l'apostrophe initiale, qui inscrit le disparu comme interlocuteur de l'ensemble du texte.

15 A cet égard, il paraît important de souligner la corrélation qui existe entre la centralité de l'interpellation et l'intensité de la relation interpersonnelle : on constate en effet que plus la proximité est forte, plus la nécrologie est «interpellative». Cette proximité peut concerner indifféremment le lien entre le locuteur et le disparu (c'est le cas dans les trois exemples ci-dessus, 3,7 et 8 , où le nécrologue était un proche du défunt), ou le lien entre le locuteur et les lecteurs (on trouve plus d'interpellations dans les journaux à diffusion restreinte et ciblée : ainsi près de la moitié des occurrences d'interpellation du corpus proviennent de Présent, alors que ce journal compte pour moins de $5 \%$ du volume du corpus). 


\section{Interpellation et double adresse}

16 Nous allons maintenant voir en quoi ces interpellations dans les nécrologies permettent de redéfinir les contours de la notion d'interpellation. Il s'agit donc, à la lumière du corpus, de contribuer à construire le concept linguistique d'interpellation.

\subsection{Double énonciation}

17 Commençons par repartir d'une définition grammaticale, celle non pas de l'interpellation (souvent délaissée par les théories linguistiques), mais de l'apostrophe (ce qui est pertinent dans la mesure où l'apostrophe est le principal phénomène interpellatif rencontré dans les nécrologies). Selon Riegel Pellat Rioul (1994), « l'apostrophe désigne la personne à qui s'adresse le locuteur; celui-ci sélectionne ainsi explicitement dans son discours le destinataire de son message " (464). C'est sur cette notion de destinataire (destinataire de l'interpellation, destinataire du message) que je souhaiterais revenir ici : je voudrais montrer que le destinataire du discours ne se superpose pas nécessairement avec l'interpelé.

18 Pour ce faire, il est nécessaire de revenir brièvement sur la scène énonciative de la nécrologie. Elle présente un emboîtement des circuits énonciatifs, caractéristique de ce qu'on appelle la double énonciation: le locuteur destine son discours au disparu (par le biais de l'interpellation, qui permet de sélectionner l'allocutaire auquel on s'adresse, même si celui-ci, dans le cas de la nécrologie, est «hors-jeu » en quelque sorte, n'étant pas en position de se muer en locuteur), mais également aux lecteurs du journal (qui sont donc une seconde catégorie de destinataires, et même de véritables coénonciateurs, comme on le verra plus tard). L'exemple suivant (il s'agit des premiers mots de la nécrologie de Georges-Paul Wagner dans Présent) témoigne de cette superposition des deux plans d'interlocution, matérialisée par une hésitation sur le destinataire ciblé :

(9) «Cher Maître... Il nous manquera. Terriblement.» [SMITS Jeanne, «Le mouvement national est en deuil », Présent, 13/06/06:1].

Dans cet exemple, mais aussi de façon générale, ces deux destinataires distincts sont tous les deux ratifiés, mais par des procédés différents. Pour le lecteur, les marques de ratification sont tacites, liées à la scène d'énonciation (le support journalistique place traditionnellement l'interaction entre le journaliste et son public). Pour le disparu, les indices de ratification sont explicites, constitués par les cas d'interpellation que nous étudions ici, qui lui donnent le statut d'allocutaire (à cet égard, il est intéressant de souligner que les cas d'interpellation du lecteur sont anecdotiques et beaucoup moins nombreux que les interpellations du disparu).

Étant donné que le discours nécrologique, dans son ensemble, s'adresse donc à deux instances différentes, on peut légitimement se demander si au niveau des séquences interpellatives, la cible réelle du discours est bien celle qui est revendiquée. Autrement dit : les interpellations sont-elles réellement destinées à l'interpelé ? Dans certains cas, il est manifeste que la séquence interpellative vise véritablement le disparu : c'est le cas des salutations ou des remerciements. En revanche, dans d'autres cas, c'est plus contestable. Reprenons ici l'exemple 3, la nécrologie de François Maurin dans l'Humanité. Ici, le destinataire avoué (le disparu, visé par l'interpellation) connaît déjà ce que le locuteur raconte (puisqu'il s'agit de sa vie). On peut donc supposer que cette séquence, en 
apparence adressée au disparu, est en réalité destinée au lecteur, seule instance énonciative pour laquelle elle est informative. L'exemple 7 présente les mêmes caractéristiques : il s'agit d'un récit de vie énoncé à la deuxième personne. Un indice supplémentaire permet ici d'affirmer que le lecteur est la cible véritable de cet énoncé, la présence $\mathrm{du}$ " on ». Ainsi, bien que l'interpellation nous présente ces énoncés comme adressés au disparu, d'autres indices, plus subtils, permettent de réinstaurer le lecteur comme destinataire véritable. Il s'agit donc ici d'un trope communicationnel, au sens que lui donne Catherine Kerbrat-Orecchioni (2002), c'est-à-dire un «décalage entre le destinataire apparent (l'adressé principal) et le destinataire réel (la cible)» (26). Nous verrons plus tard le rôle que cette mise en spectacle de l'interpellation, adressée au disparu mais destinée aux lecteurs, peut jouer dans les nécrologies.

\subsection{Rôle des tiers dans l'interpellation}

21 Je souhaiterais élargir ces quelques remarques, formulées à partir d'exemples puisés uniquement dans les nécrologies, à l'interpellation en général. On pourrait en effet objecter que l'interpellation en contexte nécrologique entre dans le cadre de l'apostrophe rhétorique, qui consiste à se détourner de son interlocuteur principal pour s'adresser à un destinataire absent ou fictif. Il y aurait donc nécessairement trope communicationnel, dans la mesure où l'adresse est feinte. Toutefois, il me semble bien plus fructueux de considérer les choses sous un autre angle et de ne pas analyser séparément apostrophe rhétorique et apostrophe interpelante : en effet, ce trope communicationnel interpellatif existe même en dehors des cas d'apostrophe rhétorique, dès lors qu'il y a double adresse. Il s'agit donc ici de montrer que la cible véritable de l'interpellation n'est pas toujours (ou du moins, pas uniquement) l'interpelé, et que l'interpellation ne consiste pas simplement, comme c'est encore couramment admis, à sélectionner son interlocuteur au sein d'un public plus large.

En dehors des situations dilogales (qui sont marginales par rapport à l'ensemble des interactions réelles, orales ou écrites), l'interpellation est un procédé qui met en jeu trois instances : le locuteur, l'interpelé, et le public «tiers » (cette désignation n'étant pas satisfaisante, les tiers étant habituellement exclus de la situation de la communication, ce qui n'est précisément pas le cas ici). Si l'implication des deux premières instances dans le processus d'interpellation est bien connu, il semble nécessaire de prendre aussi en compte les destinataires non interpelés, qui sont aussi (voire surtout) concernés par l'interpellation.

L'interpellation aurait donc dès lors une double visée illocutoire, d'une part sur l'interpelé, et d'autre part sur le public tiers, spectateur de l'interpellation, à qui elle s'adresse donc indirectement. C'est par exemple le cas de l'interpellation en contexte scolaire d'un élève par un professeur : l'interpellation s'adresse tout d'abord à l'élève afin de le rappeler à l'ordre. Mais ce faisant, l'enseignant manifeste également sa légitimité aux yeux de tous, ce qui a généralement pour effet de calmer l'ensemble de la classe. Cette double visée illocutoire se retrouve également dans les effets de l'interpellation d'un député à l'Assemblée Nationale : il peut s'agir tout d'abord de ridiculiser l'autre, mais également de ressouder son propre groupe parlementaire, à qui on s'adresse indirectement (l'effet perlocutoire de ce type d'interpellation peut être mesuré par les applaudissements qui ponctuent généralement ce genre d'interpellation). 
Cette interpellation «non-adressante " en quelque sorte peut être illustrée par des formes inédites d'interpellation, non répertoriées dans les grammaires. Il s'agit de formes d'interpellation que l'on pourrait qualifier d'impersonnelles, comme dans les exemples suivants :

(10) « Que Robert Parienté sache pourtant qu'il a aussi suscité en nous la plus vive admiration, bien au-delà de son exemplaire carrière professionnelle.» [DROUSSENT Claude, « Un exemple », L'Équipe, 29/05/06:2]

(11) «Les érudits aiment le sport, parfois, et savent pourquoi. Robert Parienté n'était pas le moins important. Qu'il trouve ici l'expression de notre modeste gratitude. » [DUCOIN Jean-Emmanuel, "Robert Parienté, homme de pointes», L'Humanité, 30/05/06:16]

(12) « C'est à la présence toujours vivante en moi du résistant que fut mon père et des valeurs qu'il défendait que j'adresse aujourd'hui mon salut.» [BERNARD Dominique, «Jean Bernard », Le Figaro, 27/04/06: 14]

Dans ces trois cas, les interpellations peuvent être dites impersonnelles dans la mesure où elles sont formulées à la troisième personne, ce qui semble de prime abord incompatible avec le fonctionnement de l'interpellation. Toutefois, même si ces formes se situent à la charnière du phénomène interpellatif, elles s'apparentent bel et bien à l'interpellation telle qu'on l'a redéfinie ici.

\section{Fonction de l'interpellation du disparu}

Après ce détour théorique, qui aura, je l'espère, permis d'éclaircir la notion d'interpellation, il est temps de revenir aux nécrologies, pour déterminer les fonctions remplies par l'interpellation du disparu. Dans son traité sur Les figures du discours (1977), Fontanier suggère que l'apostrophe révèle "le sentiment excité dans le cœur jusqu'à éclater et se répandre au dehors" (372). Certes on ne peut pas nier cette composante émotionnelle, qui joue un rôle fondamental dans les nécrologies, du fait du contexte, toutefois la fonction de l'interpellation est plus vaste : il s'agit de quelque chose de plus essentiel qu'une simple mobilisation de l'émotion.

\subsection{Redonner corps au défunt}

J'ai déjà souligné que la nécrologie débordait du cadre de l'apostrophe rhétorique, dans laquelle l'adresse est feinte et n'a donc pas à proprement parler de valeur interpellative. C'est très différent dans le cas de la nécrologie, qui apostrophe certes un être absent, mais dans le but de le présentifier, en le convoquant par le biais de la parole. L'interpellation est en effet le procédé qui permet de faire passer le disparu du statut de non-personne à celui de personne. En effet, « lorsqu'elle est employée pour référer à un être humain, la non-personne le met en absence, instaure avec lui une distance importante, une rupture » (Détrie Siblot Vérine, 2001, 24). L'interpellation du disparu permet précisément de renverser ce mouvement et de "mettre en présence », de re-présenter une entité rendue absente par la force des choses.

La nécrologie rend ainsi au disparu son statut de partenaire de l'énonciation selon deux mouvements différents. D'une part, l'interpellation permet de rendre l'absent présent au travers du verbe, non seulement par son évocation dans le contenu du discours, mais aussi par sa convocation, dans la forme même qu'adopte le discours. D'autre part, on peut observer des phénomènes qui relèvent de la prosopopée, figure par laquelle on fait parler 
l'absent : on constate en effet dans les nécrologies une propension à citer le disparu (paroles rapportées intégrées à l'article, morceaux choisis de l'œuvre prenant place dans une hyperstructure textuelle...). Ainsi la nécrologie vise, par ces artifices, à ressusciter le mort, à lui redonner corps, à l'animer par le biais du discours.

\subsection{Créer une communauté discursive} par le biais du discours. En effet, le contexte fait que la nécrologie est un texte monologal mais qui comporte un grand besoin de dialogisme. Or la nécrologie est saturée de dimension interactionnelle, entre autres grâce à l'interpellation du disparu. Le discours circule en effet entre les différentes instances énonciatives, comme en témoignent les exemples suivants :

(13) «Chers amis de Présent, j’ai la tristesse de vous annoncer la mort de mon père, ce dimanche 11 juin 2006, à 11 heures. » [WAGNER François, «Chers amis de Présent ", Présent, 13/06/06:1]

(7) «Mon cher Georges-Paul. J'ai peine à croire que j'écris ces lignes et que vous n'êtes plus là. » [PARMENTIER Caroline, « Georges-Paul », Présent, 13/06/06:1]

(14) «Georges-Paul Wagner n'a pas fait ses adieux à Présent, car il a été avec nous sur le front depuis le début, et jusqu'au bout. [...] Nous publions ici la lettre de Georges-Paul Wagner, car elle résume en quelque sorte l'héritage qu'il laisse à ses compagnons d'armes. » [WAGNER Georges-Paul, «Une dernière lettre... », Présent : $16 / 06 / 06: 2]$

Ces trois extraits proviennent d'articles parus dans Présent à l'occasion de la mort de Georges-Paul Wagner. Un seul décès a donc donné lieu à une lettre aux lecteurs écrite par le fils du défunt (exemple 13), une lettre adressée au défunt par une journaliste (exemple 7) et une publication posthume de la lettre du défunt, destinée aux lecteurs (exemple 14). Ce n'est pas un hasard si le dialogisme pénètre à ce point la nécrologie, au moment où la mort instaure le silence dans la sphère discursive et coupe les liens.

31 L'exemple suivant illustre cette circulation de la parole à tous les niveaux, les proches du disparu accédant au statut d'intermédiaire discursif vers le défunt :

(15) «Gioccante, Guillaume, Frédérique et toute la famille et les amis qui vous entourent en ce jour de grande tristesse, recevez le témoignage de notre immense affection et dites au grand Jean-Pierre que nous ne l'oublierons jamais. » [ESCANDE Philippe, «Jean-Pierre Neu », Les Échos, 17/05/06 : 10]

Si le récit est saturé de cette dimension interactionnelle, manifestée notamment par les phénomènes d'interpellation et de double adresse, c'est pour ressouder la communauté, conformément au rôle épidictique traditionnellement dévolu à la nécrologie. Ceci est d'autant plus vrai que l'interpellation est souvent utilisée comme lieu de transmission des valeurs partagées par la communauté discursive qui se recrée, comme en témoignent les deux exemples suivants, déjà analysés précédemment :

(6) «À Dieu, Devos! Que votre verbe scintillant retentisse désormais, par-delà les étoiles, au paradis des anges. » [Présent, 17/06/06:2]

(1) «Je t'aimais beaucoup, François, mon camarade des salles obscures.» [L'Humanité, 15/05/06 : 22]

Dans l'exemple 6, le choix orthographique particulier sur «à Dieu ", habituellement écrit en un seul mot, présuppose l'existence d'une vie après la mort, soulignant donc l'orientation chrétienne revendiquée par le journal. Dans l'exemple 1, l'apostrophe en «camarade» est révélatrice du positionnement communiste du journal. Ainsi, non 
seulement l'interpellation permet de ressouder la communauté, mais elle peut aussi être destinée à rappeler les valeurs qui la fondent.

L'interpellation est donc un des procédés qui permettent de recréer au sein du discours la communauté de valeurs qui rassemble le disparu, le journal et ses lecteurs. En effet, le trope communicationnel sur lequel repose l'interpellation en contexte nécrologique permet de «mettre en spectacle la coénonciation » (Détrie, 2006, 110), la connivence entre l'interpellateur et l'interpelé. Autrement dit, ce que le journaliste donne à voir au lecteur, ce n'est pas le récit de vie, mais l'interpellation elle-même, signe de la proximité qu'entretient le journaliste avec le disparu et creuset de la renaissance de la communauté.

Par ailleurs, au terme de cette étude, il semble nécessaire de souligner que l'interpellation est une notion complexe, polymorphe : il est temps de lui rendre pleinement son rôle, qui dépasse la portion congrue à laquelle elle était jusqu'alors réduite en sciences du langage. L'interpellation étant un acte foncièrement énonciatif, il parait essentiel de l'analyser non pas à partir de critères morphosyntaxiques (ce qui est aujourd'hui souvent le cas: l'analyse de l'interpellation se cantonne généralement à l'étude de la fonction syntaxique de l'apostrophe et des différentes désignations employées), mais bien à partir de critères énonciatifs, notamment la visée illocutoire de l'interpellation et ses variations en fonction du cadre participatif. On gagnerait certainement à étudier l'interpellation à la lumière des développements récents en linguistique de l'énonciation, qui remettent en cause la bipolarité de l'énonciation : la prise en compte de l'hétérogénéité énonciative, qu'elle concerne le destinataire (phénomène sur lequel je me suis penchée ici) ou l'émetteur (phénomène qui ne rentrait pas ici dans mes préoccupations, compte tenu du cadre réduit nécessairement imposé à cet article, mais pendant du phénomène précédent) serait sans aucun doute une approche fructueuse, que j'espère pouvoir mener à bien dans un avenir proche afin de confirmer et d'approfondir ces premières conclusions et de proposer une analyse plus systématique du fonctionnement discursif et pragmatique de l'interpellation.

\section{BIBLIOGRAPHIE}

Benveniste É. (1974). Problèmes de linguistique générale II. Paris : Gallimard.

Bres J., Haillet P.P., Mellet S., Rosier L., \& Nolke H. (Eds.). (2005). Dialogisme et polyphonie : approches linguistiques. Bruxelles : Duculot.

Charaudeau P., \& Maingueneau D. (Eds.). (2002). Dictionnaire d'analyse du discours. Paris : Armand Colin.

Denis D., \& Sancier-Chateau A. (1994). Grammaire du français. Paris : Livre de Poche.

Détrie C. (2006). De la non-personne à la personne : l'apostrophe nominale. CNRS.

Détrie C., Siblot P., \& Verine B., (Eds.) (2001). Termes et concepts pour l'analyse du discours : une approche praxématique, Paris : Champion. 
Fontanier P. (1977 [1821]). Les figures du discours, Paris : Flammarion.

Fromilhague C. (1995). Les figures de style. Paris : Nathan.

Gary-Prieur M.N. (1994). Grammaire du nom propre. Paris : Puf.

Goffman E. (1987 [1981]). Façons de parler. Paris : Minuit.

Jacquin G. (Ed.). (2003). Le récit de la mort. Écriture et histoire. Rennes : PUR.

Jonasson K. (1994). Le nom propre. Constructions et interprétations. Louvain-la-Neuve : Duculot.

Kerbrat-Orecchioni C. (2002). « Double adresse et récepteur multiple ». In J. Siess \& G. Valency

(Eds.), La double adresse (pp 15-40). Paris : L'Harmattan : 15-40.

Lits M. (Ed.). (1993). La peur, la mort et les médias. Bruxelles : ÉVO Communication.

Pennec S. (Ed.). (2004). Des vivants et des morts : des constructions de la « bonne mort ». Brest :

Université de Bretagne occidentale.

Rabatel A. (in press). « La question du destinataire au théâtre ». In C. Despierre \& F. Fix (Eds.), À qui parle-t-on? Le destinataire dans le théâtre vivant français. Dijon : Éditions universitaires de Dijon.

Riegel M., Pellat J.C., \& Rioul R. (1994). Grammaire méthodique du français. Paris : PUF.

Schnedecker C. (1997). Nom propre et chaînes de référence. Metz : CELTED.

Siess J. \& Valency G. (Eds.). (2002). La double adresse. Paris : L'Harmattan.

\section{RÉSUMÉS}

Cet article se veut une investigation du fonctionnement discursif et pragmatique de l'interpellation du disparu dans les nécrologies de presse, phénomène que l'on ne s'attendrait pas naturellement à y rencontrer, l'interpelé étant, par la force du contexte, exclu de la situation de communication. L'objectif poursuivi est double : il s'agit tout d'abord de déterminer le rôle tenu par l'interpellation en contexte nécrologique : nous verrons que loin d'être une originalité anecdotique, les interpellations du disparu visent à redonner corps au défunt absent, afin de ressouder la communauté, au moment où le silence la guette. Par ailleurs, cet article veut également se donner les moyens de redéfinir les contours de la notion d'interpellation, notamment en l'inscrivant dans le cadre de la théorie de l'énonciation : nous verrons que l'interpellation a la plupart du temps un double destinataire, l'allocutaire interpelé, sélectionné par la formule interpellative, mais également le public tiers, auquel le discours ne s'adresse pas, mais à qui il est malgré tout destiné, par un phénomène de trope communicationnel.

This article deals with the discursive and pragmatic functioning of the direct address to the deceased in the obituaries appearing in French press. This process may seem surprising in this genre of discourse, insofar as the person who is being addressed is inevitably excluded of the communicative situation, due to the context. This article has a dual purpose: first, it identifies the part played by direct address to the deceased in the obituary, proving that it aims at reembodying the dead person, in order to bind the community, at the very moment it is threatened by silence. Furthermore, this article intends to redefine the contours of the notion of direct address, in particular within the frame of enunciation theory: direct address has most of the time two recipients, the addressee, which is namely mentioned in the term of address, and the audience, whom the discourse is not addressed, but which is, by means of a communicative trope, the real intended recipient. 
INDEX

Mots-clés : destinataire, double énonciation, mort, nécrologie

Keywords : addressee, death, direct address, double enunciation, obituary

\section{AUTEUR}

MARIE-LAURE FLOREA

Université de Lyon 2 